\title{
Preservation of quantum correlation between separated nitrogen-vacancy centers embedded in photonic crystal cavities
}

\author{
W. L. Yang ${ }^{1,3}$, Jun-Hong $\mathrm{An}^{2,3},{ }^{*}$ Chengjie Zhang ${ }^{3}$, M. Feng ${ }^{1}{ }^{\dagger}$ and C. H. Oh ${ }^{3 \ddagger}$ \\ 1 State Key Laboratory of Magnetic Resonance and Atomic and Molecular Physics, \\ Wuhan Institute of Physics and Mathematics, Chinese Academy of Sciences, Wuhan 430071, China \\ 2 Center for Interdisciplinary Studies, Lanzhou University, Lanzhou 730000, China and \\ 3 Centre for Quantum Technologies and Department of Physics, \\ National University of Singapore, Singapore 117543, Singapore
}

\begin{abstract}
We investigate the non-Markovian dynamics of quantum correlation between two initially entangled nitrogen-vacancy centers (NVC) embedded in photonic crystal cavities (PCC). We find that a finite quantum correlation is preserved even asymptotically when the transition frequency of the NVC is within the band gap of the PCC, which is quantitatively different from the result of approaching zero under the Born-Markovian approximation. In addition, once the transition frequency of NVC is far beyond the bad gap of the PCC, the quantum correlation initially prepared in NVC will be fully transferred to the reservoirs in the long-time limit. Our result reveals that the interplay between the non-Markovian effect of the structured reservoirs and the existence of emitter-field bound state plays an essential role in such quantum correlation preservation. This feature may open new perspectives for devising active decoherence-immune solid-state optical devices.
\end{abstract}

PACS numbers: 42.50.Pq, 37.30.+i, 03.67.Bg, 76.30.Mi

\section{INTRODUCTION}

Recently, decoherence control and non-Markovian dynamics due to strong backaction from the environment have attracted much attention in practical implementation of nanoscale solid-state quantum information processing (QIP) both theoretically [1-4] and experimentally [5-9]. It is widely believed that the unavoidable interaction with Markovian environment results in decoherence effect and loss of quantum correlation, so the quantum correlation preservation $(\mathrm{QCP})$ in quantum systems has become a critical challenge in quantum computation technologies. Previous studies have shown that long-time quantum entanglement protection can be realized by engineering the structured environment such as photonic band gap (PBG) materials, which has periodic dielectric structures exhibiting a range of frequencies with electromagnetic wave propagation forbidden [10]. This novel property mostly arises from the specific structure of PBG environment [11], which leads to strong emitter-field correlation and formation of emitter-field bound states (EFBS) [12] with the spontaneous emission being greatly inhibited [13]. Experimentally, the nonMarkovian effect of the emitter in the PBG reservoir has been directly observed in several kinds of nanoscale cavities [7-9].

In this Letter, we focus on the issue of decoherence suppression in solid-state systems consisting of diamond nitrogen-vacancy centers (NVCs) [14] and planar photonic crystal cavities (PCC) [15]. The NVC is an attrac-

\footnotetext{
*Electronic address: anjhong@lzu.edu.cn

$\dagger$ Electronic address: mangfeng@wipm.ac.cn

${ }^{\ddagger}$ Electronic address: phyohch@nus.edu.sg
}

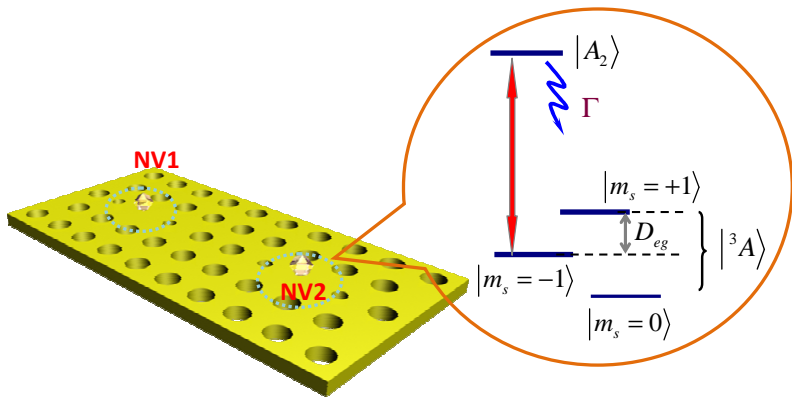

FIG. 1: (Color online) The composite NV-PCC system consists of a planar PCC and two identical NVCs in diamond nanocrystals. The inset shows the level structure of a NVC, where the electronic ground state $\left.\left.\right|^{3} A\right\rangle$ is a spin triplet state, and $D_{e g}=\gamma_{e} B_{0}$ is the level splitting induced by an external magnetic field $B_{0}$ with $\gamma_{e}$ the electron gyromagnetic ratio. The red arrow denotes the coupling between NVC and PCC. $\Gamma$ is the spontaneous decay rate of the excited state $\left|A_{2}\right\rangle$.

tive spin qubit since it exhibits a unique combination of robust room-temperature spin coherence [16] and efficient optical addressability, controllability, and readout [17-20]. Furthermore, the planar PCC with high $Q$ factor (i.e., $Q>1 \times 10^{6}$ even up to $2 \times 10^{7}$ in the optimized structure $[21,22])$ and mode volumes comparable to a cubicoptical wavelength [23] can strongly confine photons in a tiny space of optical-wavelength dimension within a PBG structure. Numerous experiments have successively demonstrated the strong coupling between NVCs and the modes of silicon nitride PCC [24], gallium phosphide PCC [21, 23, 25], and PCC in monocrystalline diamond [26, 27], respectively. These advances imply that the conventional Born-Markovian description to the NVC decoherence induced by the PPC is not applicable any- 
more.

In the present work we investigate the non-Markovian dynamics of quantum correlation between two initially entangled NVCs, each of which is embedded in a nanocavity in the planar PCC coupled to the radiation fields initially in vacuum states. Characterizing the quantum correlation by both quantum discord (QD) [28] and entanglement of formation (EoF) [29], we find that, if the transition frequency of the NVC is fully within the band gap of the PCC, a finite quantum correlation is preserved due to the interplay between the non-Markovian effect of the structured reservoirs and the existence of EFBS. Otherwise, no QCP can be observed, and there only exists the quantum correlation between the two independent reservoirs in the long-time limit. The essential condition for realizing this QCP is explicitly given. These results would be useful for experimental exploration of non-Markovian features in spin-based quantum system.

\section{THE MODEL AND HAMILTONIAN}

Now we focus on two noninteracting NVCs (NV1 and NV2) coupled, respectively, to two uncorrelated vacuum reservoirs $R_{1}$ and $R_{2}$, namely, two nanocavities in a planar PCC, as shown in Fig. 1. Each NVC is negatively charged with two unpaired electrons located near the vacancy, usually treated as electron spin-1. In our scheme, the PCC modes with $\sigma^{+}$polarization are coupled to the transition from the NVC's ground state sublevel $|-\rangle=\left|{ }^{3} A, m_{s}=-1\right\rangle$ to one of the excited states $|+\rangle=\left|A_{2}\right\rangle=\left(\left|E_{-}, m_{s}=+1\right\rangle+\left|E_{+}, m_{s}=-1\right\rangle\right) / \sqrt{2}[20]$. Due to the dynamical independence between the two local subsystems, we can first solve the individual subsystem, and then apply the result obtained to the double case. Each subsystem (i.e., a NVC plus a radiation field propagating in the $\mathrm{PCC}$ ) is governed by [30]

$$
\hat{H}=\omega_{0} \hat{\sigma}_{+} \hat{\sigma}_{-}+\sum_{k} \omega_{k} \hat{a}_{k}^{\dagger} \hat{a}_{k}+\sum_{k}\left(g_{k} \hat{\sigma}_{+} \hat{a}_{k}+h . c .\right),
$$

where $\hat{\sigma}_{ \pm}$and $\omega_{0}$ are the inversion operator and transition frequency of the NVC, and $\hat{a}_{k}^{\dagger}\left(\hat{a}_{k}\right)$ are the creation (annihilation) operators of the $k$-th mode of the reservoir. The coupling strength between the NVC and the reservoir is denoted by $g_{k}=\omega_{0} \tilde{\mathbf{d}} \cdot \tilde{\mathbf{e}}_{k} / \sqrt{2 \epsilon_{0} \omega_{k} V_{0}}$ [31], where $\tilde{\mathbf{e}}_{k}$ and $V_{0}$ are the unit polarization vector and the normalization volume of the radiation field, $\tilde{\mathbf{d}}$ is the dipole moment of the NVC, and $\epsilon_{0}$ is the free space permittivity. Here the specific periodic structure of the PCC causes a band-gap dispersion relation to the field [32].

$$
\omega_{k}=\omega_{c}+A\left(k-k_{0}\right)^{2},
$$

where $A \simeq \omega_{c} / k_{0}^{2}$ with $k_{0} \simeq \omega_{c} / c$ being a specific wave vector with respect to the point-group symmetry of the PCC, and $\omega_{c}$ is the dip of the band frequency. Note that Eq. (2) describes well our 2D PPC structure, where the 2D square lattice is formed by cylinders (see Fig. 1).
Here the two orthogonal directions are equivalent and the dispersion relation is the same for both.


FIG. 2: (Color online) Time evolution of the quantum correlations between NV1 and NV2 calculated by QD (solid-line) and EoF (dashed-line), where (a) $\alpha=1 / \sqrt{2}$, and $\omega_{0}=\omega_{c} / 10$; (b) $\alpha=0.2$, and $\omega_{0}=\omega_{c} / 10$; (c) $\alpha=0.3$, and $\omega_{0}=\omega_{c} / 10$; (d) $\alpha=1 / \sqrt{2}$, and $\omega_{0}=10 \omega_{c} ;$ (e) $\alpha=0.2$, and $\omega_{0}=10 \omega_{c}$; (f) $\alpha=0.3$, and $\omega_{0}=10 \omega_{c}$. The parameter $\eta=0.2$ is used.

\section{THE DYNAMICS OF QUANTUM CORRELATIONS}

We firstly study the time evolution of a single NVC prepared initially in the excited state $|+\rangle$ influenced by the reservoir. The state of the system evolves as

$$
|\psi(t)\rangle=b(t)\left|+,\left\{0_{k}\right\}\right\rangle+\sum_{k=0}^{\infty} b_{k}(t)\left|-,\left\{1_{k}\right\}\right\rangle,
$$

where $\left|\left\{1_{k}\right\}\right\rangle$ is the single-photon state in $k$-th mode of the reservoir. The amplitude $b(t)$ at any time satisfies following integro-differential equation

$$
\dot{b}(t)+i \omega_{0} b(t)+\int_{0}^{t} f(t-\tau) b(\tau) d \tau=0,
$$

where the correlation function is $f(t-\tau)=$ $\eta \int \frac{k^{2} c^{3}}{\omega_{k}} e^{-i \omega_{k}(t-\tau)} d k$. The non-Markovian memory effect of the structured reservoir has been registered selfconsistently in the kernel function $f(t-\tau)$ in Eq. (4). Going back to the double-NVC case, the master equation of NVCs can be derived by tracing over the environmental degrees of freedom from Eq. (3) [1],

$$
\begin{aligned}
\dot{\rho}(t)= & \sum_{n=1}^{2}\left\{-i \Omega(t)\left[\sigma_{+}^{n} \sigma_{-}^{n}, \rho(t)\right]+\gamma(t)\left[2 \sigma_{-}^{n} \rho(t) \sigma_{+}^{n}\right.\right. \\
& \left.\left.-\sigma_{+}^{n} \sigma_{-}^{n} \rho(t)-\rho(t) \sigma_{+}^{n} \sigma_{-}^{n}\right]\right\}
\end{aligned}
$$

where $\Omega(t)=-\operatorname{Im}\left[\frac{\dot{b}(t)}{b(t)}\right]$ and $\gamma(t)=-\operatorname{Re}\left[\frac{\dot{b}(t)}{b(t)}\right]$ denote Lamb shifted frequency and decay rate of the NVCs, respectively. If $f(t-\tau)$ in Eq. (4) is replaced by a delta 
function, then Eq. (5) recovers the conventional master equation under Born-Markovian approximation [33].

Starting from the initial state of the whole system as $|\Psi(0)\rangle=(\alpha|-,-\rangle+\beta|+,+\rangle)\left|\left\{0_{k}\right\}\right\rangle_{r_{1}}\left|\left\{0_{k}\right\}\right\rangle_{r_{2}}[34]$, we can obtain the time-dependent state as

$$
|\Psi(t)\rangle=\alpha\left|-,\left\{0_{k}\right\}\right\rangle_{1}\left|-,\left\{0_{k}\right\}\right\rangle_{2}+\beta|\psi(t)\rangle_{1}|\psi(t)\rangle_{2},
$$

where Eq. (3) has been rewritten as $|\psi(t)\rangle=$ $b(t)|+\rangle|\overline{0}\rangle_{r}+\tilde{b}(t)|-\rangle|\overline{1}\rangle_{r}$ with the collective states of the reservoir defined as $|\overline{0}\rangle_{r}=\left|\left\{0_{k}\right\}\right\rangle$ and $|\overline{1}\rangle_{r}=$ $\frac{1}{b(t)} \sum_{k} b_{k}(t)\left|1_{k}\right\rangle$ and $\tilde{b}(t)=\sqrt{1-|b(t)|^{2}}[36]$. From Eq. (6) the reduced density matrix $\rho_{N_{1} N_{2}}$ for the subsystem NV1-NV2 can be obtained by tracing over the degrees of freedom of the two reservoirs,

$$
\rho_{N_{1} N_{2}}(t)=\left(\begin{array}{cccc}
|\beta|^{2}|b(t)|^{4} & 0 & 0 & \beta \alpha^{*} b(t)^{2} \\
0 & p & 0 & 0 \\
0 & 0 & p & 0 \\
\beta^{*} \alpha b^{*}(t)^{2} & 0 & 0 & q
\end{array}\right)
$$

with $p=|\beta b(t)|^{2} \tilde{b}(t)^{2}$ and $q=1-|\beta|^{2}|b(t)|^{4}-2 p$. Similarly, the corresponding reduced density matrices can be obtained for other subsystems like reservoir1-reservoir2 $\left(r_{1} r_{2}\right)$ and NVC-reservoir $\left(N_{1} r_{1}, N_{1} r_{2}, N_{2} r_{1}, N_{2} r_{2}\right)$.

The quantum correlations can be quantified by EoF [29] and QD [28, 37]. The former is defined as $E(\rho)=$ $H\left[\frac{1+\sqrt{1-C(\rho)^{2}}}{2}\right]$ with $H[x]=-x \log _{2} x-(1-x) \log _{2}(1-x)$ and $C(\rho)=\max \left\{0, \sqrt{\lambda_{1}}-\sqrt{\lambda_{2}}-\sqrt{\lambda_{3}}-\sqrt{\lambda_{4}}\right\}$, where the decreasing-order-arranged quantities $\lambda_{i}$ are the eigenvalues of the matrix $\rho\left(\sigma_{y}^{A} \otimes \sigma_{y}^{B}\right) \rho^{*}\left(\sigma_{y}^{A} \otimes \sigma_{y}^{B}\right)$ with $\rho^{*}$ the complex conjugation of $\rho$ and $\sigma_{y}^{A(B)}$ the Pauli matrix acting on the subsystem $A(B)$ [38]. On the other hand, $\mathrm{QD}$ is defined as the minimum difference between two ways defining mutual information $(\mathrm{MI}), Q(\rho)=I\left(\rho_{A B}\right)-$ $\max _{\left\{\Pi_{k}\right\}} I\left(\rho_{A} \mid\left\{\Pi_{k}\right\}\right)$. Here $I\left(\rho_{A B}\right)=S\left(\rho_{A}\right)+S\left(\rho_{B}\right)-$ $S\left(\rho_{A B}\right)$ is the quantum MI and $\max _{\left\{\Pi_{k}\right\}} I\left(\rho_{A} \mid\left\{\Pi_{k}\right\}\right)=$ $\max _{\left\{\Pi_{k}\right\}}\left[S\left(\rho_{A}\right)-\sum_{k} p_{k} S\left(\rho_{A}^{k} \mid \Pi_{k}\right)\right]$ is the maximal MI when a measurement is performed on subsystem $B$ [28], where $S(\cdot)$ is the von Neumann entropy, $\left\{\Pi_{k}\right\}$ is a completely positive-operator-valued measure on the subsystem $B$ and $p_{k}$ is the respective probabilities.

Fig. 2 presents the quantum correlation dynamics (EoF and QD) of the NV1-NV2 subsystem with different initial states, where two typical cases of $\omega_{0}<\omega_{c}$ and $\omega_{0} \gg \omega_{c}$ are considered, corresponding to the NVC's transition frequency within and far beyond the band gap of the PBG material, respectively. In the former case, as shown in Fig. 2(a,b,c), the correlations calculated by EoF and QD exhibit obvious oscillation due to the energy exchange between the NVC and the memory reservoir, and then the correlations approach a definite value in the long-time limit. It implies that the decay rate of the excited state $\left|A_{2}\right\rangle$ of the NVC approaches zero after some oscillations, which leads to the QCP. However, in the $\omega_{0} \gg \omega_{c}$ case, the residual correlation by EoF and QD between NV1 and NV2 vanishes in the long-time limit, although there exists transient revival in EoF [see Fig. 2(e,f)]. All the differences between these two cases indicate that the PBG plays a vital role in the quantum correlation evolution of the NVCs confined in the structured environment. We will return to this point later.
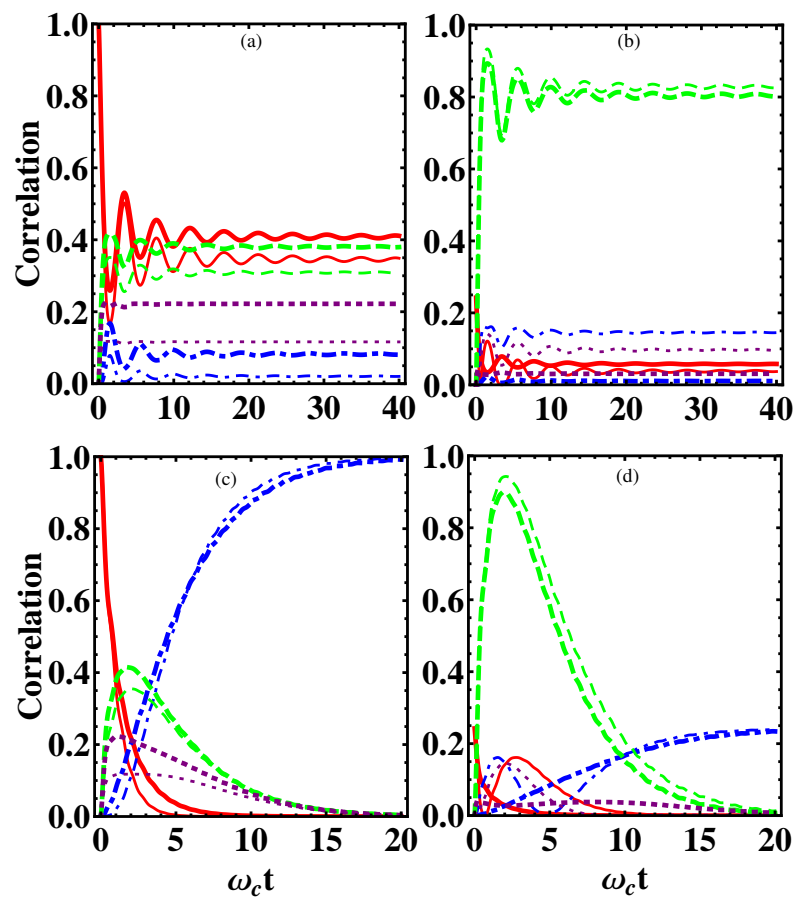

FIG. 3: (Color online) Time evolution of the quantum correlations calculated by QD (thick-line) and EoF (thin-line) for different subsystems, where the thick-solid line, thick-dotdashed line, thick-dashed line, and thick-dotted line denote the QD1, QD2, QD3, and QD4, respectively. The thin-solid line, thin-dot-dashed line, thin-dashed line, and thin-dotted line denote the Eof1, Eof2, Eof3, and Eof4, respectively. (a) $\alpha=1 / \sqrt{2}$, and $\omega_{0}=\omega_{c} / 10$; (b) $\alpha=0.2$, and $\omega_{0}=\omega_{c} / 10$; (c) $\alpha=1 / \sqrt{2}$, and $\omega_{0}=10 \omega_{c} ;$ (d) $\alpha=0.2$, and $\omega_{0}=10 \omega_{c}$. The parameter $\eta=0.2$ is used.

To get a clear picture on how the quantum correlation is distributed among different subsystems, we have calculated quantum correlations for different bipartite partitions, such as reservoir1-reservoir2, NV1-reservoir1, and NV1-reservoir2 labeled by QD2 (Eof2), QD3 (EoF3) and QD4 (EoF4), respectively, and the quantum correlations between NV1-NV2 are denoted by QD1 (EoF1). Fig. 3 shows the time evolution of EoF and QD in each subsystem under different initial states, and one can find that for each partition, the correlations denoted by EoF and QD behave similarly with obvious oscillations. Interestingly, once the two NVCs are initially prepared in maximally entangled state with $\alpha=\beta=1 / \sqrt{2}$, QD is always larger than EoF for both $\omega_{0}<\omega_{c}$ and $\omega_{0} \gg \omega_{c}$ cases, as shown in Fig. 3(a,c). The only exception is the curves for QD2 and EoF2 in Fig. 3(c) in the $\omega_{0} \gg \omega_{c}$ case, which do not obey the monogamic relation. In contrast to this feature, if the NVCs are not initially prepared in maximally entangled states, as in Fig. 3(b,d), 

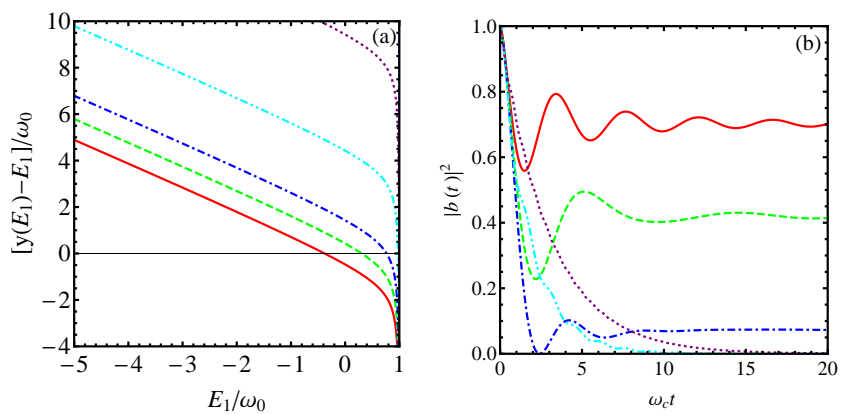

FIG. 4: (Color online) Illustration of the formation of EFBS (a) and its dynamical consequence to the excited-state population (b) when $\omega_{0}=\omega_{c} / 10$ (solid line), $\omega_{c}$ (dashed line), $2 \omega_{c}$ (dot-dashed line), $5 \omega_{c}$ (dot-dot-dashed line), and $10.0 \omega_{c}$ (dotted line). The intersect of $y\left(E_{1}\right)-E_{1}$ with the horizontal axis characterizes the formation of EFBS. $\eta=0.2$ is used.

QD is usually smaller than EoF. Furthermore, it is important to note that the QCP in each subsystem exists only for the NV's transition frequency within the band gap, as shown in Fig. 3(a,b). Another obvious feature is that the quantum correlation initially prepared between NVCs distributes between other subsystems with different weights. However, for $\omega_{0} \gg \omega_{c}$, the residual quantum correlation of QD1, QD3, and QD4 vanishes in the long-time limit, but the quantum correlations (QD2 and EoF2) between the two independent reservoirs approach their maximum values, which implies that quantum correlation initially prepared in NVCs has been fully transferred to the reservoirs, significantly different from the multi-site distribution of quantum correlation in the case of $\omega_{0}<\omega_{c}$.

\section{THE DISCUSSION AND CONCLUSION}

The Born-Markovian approximation is only valid in our case when the correlation function of the radiation field has an infinitesimal time scale, which is always valid when its density of states is a continuous and smoothly varying function in the frequency space [39]. However, the distribution function for PBG reservoir is not a monotonous function due to the existence of band edge, where the density of states varies rapidly with a rate comparable to the NVC's spontaneous emission rate [12], then it becomes non-exponential and the emission spectrum becomes non-Lorentzian. By accurately calculating the non-Markovian dynamics, we really find that the quantum correlation dynamics displays an oscillatory behavior and non-exponential tendency. Figs. 2 and 3 indicate clearly that the photon emitted through spontaneous emission goes back and forth between the NVCs and their local reservoirs. It means that the environment is not robust any more during the interaction with the quantum system, but changes at the time scale of its memory time to reach a new equilibrium state.
We attribute the mechanism of this novel QCP to the interplay between the formed EFBS and the nonMarkovian effect. Here we argue that there are two essential conditions for realizing the QCP. One is the existence of the EFBS, which provides the ability to preserve quantum correlation, and the other is the non-Markovian effect, which provides a dynamical way to preserve the quantum correlation [40]. Thus, whether the EFBS exists or not plays a key role in achieving the QCP, even if the reservoir has already shown the memory effect.

To verify this argument, we find under what condition the EFBS can be formed. The total excitation number $\hat{N}=\hat{\sigma}_{+} \hat{\sigma}_{-}+\sum_{k} \hat{a}_{k}^{\dagger} \hat{a}_{k}$ of Eq. (1) is conserved, so the Hilbert space is split into the direct sum of the subspaces with definite $N$. From the eigen equation in the $N=1$ subspace, we have the eigenvalue $E_{1}$ fulfilling

$$
y\left(E_{1}\right) \equiv \omega_{0}+\eta \int_{0}^{\infty} \frac{c^{3} k^{2}}{\left(E_{1}-\omega_{k}\right) \omega_{k}} d k=E_{1},
$$

where $\eta=\omega_{0}^{2} d^{2} / 6 \pi^{2} \epsilon_{0} c^{3}$ is a dimensionless coupling constant, and $\eta \omega_{0}$ is the natural spontaneous emission rate of the excited state $\left|A_{2}\right\rangle$ of NVC. Combining with Eq. (2), we can find that $y\left(E_{1}\right)$ is monotonically decreasing in the region $E_{1} \in\left(-\infty, \omega_{c}\right)$, i.e. in the band gap of the reservoir. It means that Eq. (8) may have one and only one solution in this region if the system parameters fulfill $y\left(\omega_{c}\right)<\omega_{c}$ [see Fig. 4(a)]. The curves when $\omega_{0}$ is smaller than or comparable with $\omega_{c}$ definitively have an intersection point with the horizontal line so that one discrete root exists for Eq. (8). We call this discrete eigenstate as the EFBS. On the other hand, no root exists in the region $\left(\omega_{c},+\infty\right)$ because it would make $y\left(E_{1}\right)$ divergent otherwise. As a stationary state of the whole system, the EFBS does not lose any quantum coherence during the time evolution, implying that the decoherence can be inhibited. Fig. 4(b) shows the dynamical consequence of the EFBS on the excited-state population of the NVC. We really find that once the EFBS is formed, the excitedstate population will stabilize to a steady value in the long-time limit.

The underlying physical picture is that during the evolution the NVC's excited level $\left|A_{2}\right\rangle$ experiences an anomalous "giant" Lamb shift and splits into doublet levels only when the NVC's transition frequency $\omega_{0}$ lies inside or near the band gap [41-43]. One of the doublet levels retains in EFBS lying within the gap, whereas the other is shifted out of the gap and exhibits resonance fluorescence. In the $\omega_{0}<\omega_{c}$ case, once the FEBS has been established, the excited-state population of the NVC in a FEBS remains constant in time because the FEBS state is actually a stationary state with a vanishing decay rate during the time evolution. The claim above can be further verified by the fact that the QCP is always absent in the $\omega_{0} \gg \omega_{c}$ case, as shown in the right panels of Fig. 2 and in the lower panels of Fig. 3, where the EFBS does not appear due to the NVC's transition frequency far beyond the upper band of the PBG. It is physically originated from the fact that the eigenstate of the Hamil- 
tonian (3) with the eigenvalues resides in a continuous band experiences an out-of-phase interference during the time evolution, which makes the excited-state population approaching zero asymptotically and causes the severe decoherence to the NV1-NV2 subsystem.

In summary, we have investigated the dynamics of quantum correlation between two initially entangled NVCs embedded, respectively, in PCC. We have shown the existence of QCP resulted from both the memory effect of the non-Markovian structured reservoir and the EFBS. With the mechanism of QCP, we expect to have a significant step toward the future full-scale quantum information processor based on the increasingly-developed nanoscale solid-state technology. It is noted that although only 2D structure of photonic crystal is taken into accounted in our work, our result could potentially be applicable to the 3D case with a more complicated PBG structure [8]. We expect that the QCP could be achieved whenever the EFBS is formed under the condition that the transition frequency of the NVC is within the band gap of the $3 \mathrm{D}$ photonic crystal. The qualitative verification to this expectation is worth to be performed by combing the explicit form of $3 \mathrm{D}$ photonic crystal structure in the future.

\section{Acknowledgements}

The authors thank Z. Y. Xu, Q. J. Tong, and Z. Q. Yin for enlightening discussions. This work is supported partially by the National Fundamental Research Program of China under Grant No. 2012CB922102, by the NNSF of China under Grants No. 11274351, No. 10974225, No. 11004226, No. 11104326, No. 11105136, No. 11175072 and No. 11204196, by the Specialized Research Fund for the Doctoral Program of Higher Education, and by the National Research Foundation and Ministry of Education, Singapore (Grant No. WBS: R-710-000-008-271).
[1] H.-P Breuer and F. Petruccione, The Theory of Open Quantum Systems (Oxford University, Oxford, 2007).

[2] P. Kaer, T. R. Nielsen, P. Lodahl, A.-P. Jauho, and J. Mørk, Phys. Rev. Lett. 104, 157401 (2010); M. Žnidarič, C. Pineda, and I. García-Mata, Phys. Rev. Lett. 107, 080404 (2011); E.-M. Laine, H.-P. Breuer, J. Piilo, C.-F. Li, and G.-C. Guo, Phys. Rev. Lett. 108, 210402 (2012); A. Carmele, A. Knorr, and F. Milde, arXiv:1203.0126.

[3] S. Maniscalco, F. Francica, R. L. Zaffino, N. L. Gullo, and F. Plastina, Phys. Rev. Lett. 100, 090503 (2008); D. Alonso and Inés de Vega, Phys. Rev. Lett. 94, 200403 (2005); J. Piilo, S. Maniscalco, K. Härkönen, and K.-A. Suominen, Phys. Rev. Lett. 100, 180402 (2008).

[4] W.-M. Zhang, P.-Y. Lo, H.-N. Xiong, M. W.-Y. Tu, and F. Nori, Phys. Rev. Lett. 109, 170402 (2012); L. Ferialdi and A. Bassi, Phys. Rev. Lett. 108, 170404 (2012); S. F. Huelga, Á. Rivas, and M. B. Plenio, Phys. Rev. Lett. 108, 160402 (2012).

[5] H. Tahara, Y. Ogawa, and F. Minami, Phys. Rev. Lett. 107, 037402 (2011); C. Galland, A. Högele, H. E. Türeci, and A. Imamoğlu, Phys. Rev. Lett. 101, 067402 (2008).

[6] B.-H. Liu, L. Li, Y.-F. Huang, C.-F. Li, G.-C. Guo, E.-M. Laine, H.-P. Breuer, and J. Piilo, Nat. Phys. 7, 931 (2011); B.-H. Liu, D.-Y. Cao, Y.-F. Huang, C.F. Li, G.-C. Guo, E.-M. Laine, H.-P. Breuer, J. Piilo, arXiv:1208.1358.

[7] K. H. Madsen, S. Ates, T. Lund-Hansen, A. Löffler, S. Reitzenstein, A. Forchel, and P. Lodahl, Phys. Rev. Lett. 106, 233601 (2011).

[8] U. Hoeppe, C. Wolff, J. Küchenmeister, J. Niegemann, M. Drescher, H. Benner, and K. Busch, Phys. Rev. Lett. 108, 043603 (2012).

[9] K. H. Madsen, P. Kaer, A. Kreiner-Møller, S. Stobbe, A. Nysteen, J. Mørk, P. Lodahl, arXiv:1205.5623.

[10] M. Fujita, S. Takahashi, Y. Tanaka, T. Asano, and S. Noda, Science 308, 1296 (2005).

[11] N. Vats and S. John, Phys. Rev. A 58, 4168 (1998).

[12] S. John, Phys. Rev. Lett. 58, 2486 (1987); S. John and
J. Wang, ibid. 64, 2418 (1990); S. John and T. Quang, ibid. 74, 3419 (1995).

[13] M. D. Leistikow, A. P. Mosk, E. Yeganegi, S. R. Huisman, A. Lagendijk, and W. L. Vos, Phys. Rev. Lett. 107, 193903 (2011); M. R. Jorgensen, J. W. Galusha, and M. H. Bartl, Phys. Rev. Lett. 107, 143902 (2011); H. Ning and P. V. Braun, Physics 4, 76 (2011).

[14] F. Jelezko, T. Gaebel, I. Popa, A. Gruber, and J. Wrachtrup, Phys. Rev. Lett. 92, 076401 (2004); D. M. Toyli, D. J. Christle, A. Alkauskas, B. B. Buckley, C. G. Van de Walle, and D. D. Awschalom, Phys. Rev. X 2, 031001 (2012).

[15] B.-S. Song, S. Noda, T. Asano, and Y. Akahane, Nat. Mater. 4, 207 (2005); J. H. Hartmann, F. G. S. L. Brandäo, and M. B. Plenio, Nat. Phys. 2, 849 (2006); D. van Oosten and L. Kuipers, Phys. Rev. A 84, 011802(R) (2011).

[16] P. C. Maurer, G. Kucsko, C. Latta, L. Jiang, N. Y. Yao, S. D. Bennett, F. Pastawski, D. Hunger, N. Chisholm, M. Markham, D. J. Twitchen, J. I. Cirac, and M. D. Lukin, Science 336, 1283 (2012); G. Balasubramanian, P. Neumann, D. Twitchen, M. Markham, R. Kolesov, N. Mizuochi, J. Isoya, J. Achard, J. Beck, J. Tissler, V. Jacques, P. R. Hemmer, F. Jelezko, and J. Wrachtrup, Nat. Mater. 8, 383 (2009).

[17] P. Neumann, J. Beck, M. Steiner, F. Rempp, H. Fedder, P. R. Hemmer, J. Wrachtrup, and F. Jelezko, Science 329, 542 (2010); B. B. Buckley, G. D. Fuchs, L. C. Bassett, and D. D. Awschalom, Science 330, 1212 (2010); L. Robledo, L. Childress, H. Bernien, B. Hensen, P. F. A. Alkemade, and R. Hanson, Nature (London) 477, 574 (2011); A. Gruber, A. Dräbenstedt, C. Tietz, L. Fleury, J. Wrachtrup, and C. von Borczyskowski, Science 276, 2012 (1997).

[18] T. van der Sar, Z. H. Wang, M. S. Blok, H. Bernien, T. H. Taminiau, D. M. Toyli, D. A. Lidar, D. D. Awschalom, R. Hanson, and V. V. Dobrovitski, Nature (London) 484, 82 (2012); P. Huang, X. Kong, N. Zhao, F. Shi, P. Wang, 
X. Rong, R.-B. Liu, and J. Du, Nat. Commun. 2, 570 (2011); M. V. Dutt, L. Childress, L. Jiang, E. Togan, J. Maze, F. Jelezko, A. S. Zibrov, P. R. Hemmer, and M. D. Lukin, Science 316, 1312 (2007).

[19] J. R. Maze, P. L. Stanwix, J. S. Hodges, S. Hong, J. M. Taylor, P. Cappellaro, L. Jiang, M. V. Gurudev Dutt, E. Togan, A. S. Zibrov, A. Yacoby, R. L. Walsworth, and M. D. Lukin, Nature (London) 455, 644 (2008); E. Togan, Y. Chu, A. Imamoğlu, and M. D. Lukin, Nature (London) 478, 497 (2011); F. Dolde, H. Fedder, M. W. Doherty, T. Nöbauer, F. Rempp, G. Balasubramanian, T. Wolf, F. Reinhard, L. C. L. Hollenberg, F. Jelezko, and J. Wrachtrup, Nat. Phys. 7, 459 (2011); G. Goldstein, P. Cappellaro, J. R. Maze, J. S. Hodges, L. Jiang, A. S. Sørensen, and M. D. Lukin, Phys. Rev. Lett. 106, 140502 (2011).

[20] E. Togan, Y. Chu, A. S. Trifonov, L. Jiang, J. R. Maze, L. Childress, M. V. G. Dutt, A. S. Sørensen, P. R. Hemmer, A. S. Zibrov, and M. D. Lukin, Nature (London) 466, 730 (2010).

[21] J. Wolters, A. W. Schell, G. Kewes, N. Nüsse, M. Schoengen, H. Döscher, T. Hannappel, B. Löchel, M. Barth, and O. Benson, Appl. Phys. Lett. 97, 141108 (2010).

[22] T. van der Sar, J. Hagemeier, W. Pfaff, E. C. Heeres, S. M. Thon, H. Kim, P. M. Petroff, T. H. Oosterkamp, D. Bouwmeester, and R. Hanson, Appl. Phys. Lett. 98, 193103 (2011).

[23] D. Englund, B. Shields, K. Rivoire, F. Hatami, J. Vučković, H. Park, and M. D. Lukin, Nano Lett. 10, 3922 (2010).

[24] M. W. McCutcheon and M. Lončar, Opt. Express 16, 19136 (2008).

[25] P. E. Barclay, K. M. Fu, C. Santori, and R. G. Beausoleil, Opt. Express 17, 9588 (2009).

[26] A. Faraon, C. Santori, Z. Huang, V. M. Acosta, and R. G. Beausolei, Phys. Rev. Lett. 109, 033604 (2012).

[27] J. Wolters, G. Kewes, A. W. Schell, N. Nüsse, M. Schoengen, B. Löchel, T. Hanke, R. Bratschitsch, A. Leitenstorfer, T. Aichele, and O. Benson, Phys. Status Solidi B 249, 918 (2012).

[28] H. Ollivier and W. Zurek, Phys. Rev. Lett. 88, 017901
(2001); L. Henderson and V. Vedral, J. Phys. A: Math. Gen. 34, 6899 (2001).

[29] C. H. Bennett, D. P. DiVincenzo, J. A. Smolin, and W. K. Wootters, Phys. Rev. A 54, 3824 (1996).

[30] M. O. Scully and M. S. Zubairy, Quantum Optics (Cambridge University Press, Cambridge, 1997).

[31] S. M. Spillane, T. J. Kippenberg, K. J. Vahala, K. W. Goh, E. Wilcut, and H. J. Kimble, Phys. Rev. A 71, 013817 (2005).

[32] S. John and T. Quang, Phys. Rev. A 50, 1764 (1994).

[33] G. S. Agarwal, Quantum Optics (Springer-Verlag, Berlin, 1974); K. Mølmer and S. Bay, Phys. Rev. A 59, 904 (1999).

[34] The preparation of initial entangled state could be completed by making the two seperated NVCs sharing a common single-excitation, which is initially prepared in a NVC. The single-excitation will ultimately tunnel into two emitters with a small/large speed in the presence of the weak/strong cavity-cavity tunneling effect during the evolution process. The relevant details can be found in the paper [35].

[35] W. L. Yang, Z. Q. Yin, Z. Y. Xu, M. Feng, and C. H. Oh, Phys. Rev. A 84, 043849 (2011).

[36] C. E. López, G. Romero, F. Lastra, E. Solano, and J. C. Retamal, Phys. Rev. Lett. 101, 080503 (2008).

[37] M. F. Cornelio, M. C. de Oliveira, and F. F. Fanchini, Phys. Rev. Lett. 107, 020502 (2011).

[38] W. K. Wootters, Phys. Rev. Lett. 80, 2245 (1998).

[39] Inés de Vega and D. Alonso, Phys. Rev. A 77, 043836 (2008).

[40] Q.-J. Tong, J.-H. An, H.-G. Luo, and C. H. Oh, Phys. Rev. A 81, 052330 (2010).

[41] M. Woldeyohannes and S. John, J. Opt. B: Quantum Semiclass. Opt. 5, R43 (2003).

[42] D. Mogilevtsev, F. Moreira, S. B. Cavalcanti, and S. Kilin, Phys. Rev. A 75, 043802 (2007).

[43] B. Bellomo, R. Lo Franco, S. Maniscalco, and G. Compagno, Phys. Rev. A 78, 060302(R) (2008); M. Al-Amri, Gao-xiang Li, R. Tan, and M. Suhail Zubairy, Phys. Rev. A 80, 022314 (2009). 